\title{
Cloning and Characterization of Allograft Inflammatory Factor-1: A Novel Macrophage Factor Identified in Rat Cardiac Allografts with Chronic Rejection
}

\author{
Ulrike Utans, Robert J. Arceci, * Yukari Yamashita, and Mary E. Russell \\ Harvard School of Public Health and Brigham and Women's Hospital, Boston, Massachusetts 02115; and *Hematology/Oncology, \\ Children's Hospital Medical Center, Cincinnati, Ohio 45229
}

\begin{abstract}
The development of arteriosclerotic lesions in the Lewis to F344 rat model of chronic cardiac rejection is characterized by macrophage adhesion to the vessel lumen and macrophage infiltration in the neointima prior to smooth muscle cell accumulation. We report the cloning and characterization of allograft inflammatory factor-1 (AIF-1), a novel cDNA that is expressed early and persistently in chronically rejecting cardiac allografts but is absent in cardiac syngrafts and host hearts. The full-length cDNA codes for a hydrophilic polypeptide of $17 \mathrm{kD}$ that contains a 12-amino acid region similar to an EF-hand (calcium-binding) domain. In cardiac allografts AIF-1 transcripts and protein localized to infiltrating mononuclear cells. Analysis of isolated cell populations confirmed that AIF-1 was selectively expressed in macrophages and neutrophils and demonstrated that AIF-1 transcripts could be upregulated by sixfold after stimulation with the $\mathbf{T}$ cell-derived cytokine IFN- $\boldsymbol{\gamma}$. Treatment with a diet deficient in essential fatty acids (which attenuates arteriosclerosis) or CTLA-4 Ig (which blocks lymphocyte activation) significantly decreased AIF-1 transcript levels. Upregulation of AIF-1 in the setting of $T$ cell activation suggests that it may play a role in macrophage activation and function. (J. Clin. Invest. 1995. 95:2954-2962.) Key words: macrophages • rejection • cardiac transplantation • arteriosclerosis $\bullet$ gene expression
\end{abstract}

\section{Introduction}

The major manifestation of chronic cardiac rejection is transplant arteriosclerosis (1-3). It is the leading cause of graft failure and retransplantation after the first postoperative year. Little is known about the pathogenesis of this diffuse, concentric intimal thickening that affects a spectrum of vessels in transplanted hearts. Because arterial lesions typically occur in donor vessels and are absent in recipient vessels, the development of arteriosclerosis appears to reflect a localized immune-mediated process. The most widely held hypothesis is that an alloimmune response involving activated inflammatory cells and vascular cells produces cytokines and growth factors that in turn mediate smooth muscle-cell migration and proliferation.

Address correspondence to Mary E. Russell, M.D., Cardiovascular Biology Laboratory, Harvard School of Public Health, 677 Hungtington Ave., Boston, MA 02115. Phone: 617-432-4996; FAX: 617-432-2980.

Received for publication 12 December 1994 and in revised form 17 February 1995.

J. Clin. Invest.

(C) The American Society for Clinical Investigation, Inc.

0021-9738/95/06/2954/09 \$2.00

Volume 95, June 1995, 2954-2962
Animal models of cardiac transplantation have provided a way to evaluate the cellular and molecular components of transplant arteriosclerosis $(4,5)$. Detailed immunocytochemical analyses of specific inflammatory cell types that are present at various times in the Lewis to F344 rat cardiac allograft model point to a key role for the macrophage $(6,7)$. Arteriosclerotic lesions in vessels from Lewis to F344 rat cardiac allografts develop in stages. The earliest (7-14 d) involves the adhesion of mononuclear cells to the vessel lumen. By day 30 an early neointima forms that is composed predominantly of infiltrating macrophages with some $\mathrm{T}$ lymphocytes. Between days 30 and $75 \alpha$ actin-positive smooth muscle cells appear in the thickened neointima interposed among the inflammatory cells. Neointimal expansion is most striking after $100 \mathrm{~d}$ at which point it is often obliterative, with a predominance of smooth muscle cells and diminishing numbers of inflammatory cells.

Recent studies completed in our lab have examined the role of macrophages in the pathogenesis of chronic rejection and have demonstrated that macrophage activation occurs within the transplanted heart in concert with increased expression of inducible effectors such as monocyte chemoattractant protein1 , inducible nitric oxide synthase, and galactose $/ N$-acetylgalactosamine macrophage lectin (8-10). Macrophage activation and diversity are regulated in part by alterations in gene expression (11). Thus, one of our goals has been to study the macrophage directly in the transplanted heart as a way of preserving the multiple cell types and stimuli that regulate its functional state. One approach has involved using differential mRNA display to identify genes whose expression increases in cardiac allografts undergoing chronic rejection in comparison with cardiac syngrafts (12). These studies resulted in the identification of a novel cDNA fragment that we termed allograft inflammatory factor-1 (AIF-1). ${ }^{1}$ The purpose of the present study was to isolate the full-length AIF-1 cDNA and characterize its expression in vivo during various stages of chronic rejection and in vitro after cytokine stimulation. We demonstrate that the T cell-derived cytokine IFN- $\gamma$ up-regulates AIF-1 expression, suggesting a possible functional role for AIF-1 in macrophage activation and function.

\section{Methods}

Lewis to F344 rat cardiac transplantation. The heterotopic abdominal cardiac transplantation samples used in this study were originally procured in experimental sets described previously $(9,13,14)$. For allogeneic combinations, F344 rats were used as recipients and Lewis rats were used as donors. For syngeneic combinations Lewis rats served as recipients and donors. At the time of harvest both the host (recipient) heart, which served as a reference exposed to the same circulation, and

1. Abbreviations used in this paper: AIF-1, allograft inflammatory factor-1; EFAD, essential fatty acid-deficient. 
the transplanted heart were collected for histologic analysis and RNA extraction.

Inflammatory cell populations. A lymphocyte-enriched cell population was obtained by purifying isolated splenocytes on nylon wool (9). Peritoneal inflammatory rat macrophages were elicited with thioglycolate medium or the bacille Calmette Guérin as described $(9,15)$. Rat bone marrow-derived macrophages were obtained by growing femoral bone marrow cells from Lewis rats in tissue culture medium containing murine macrophage colony stimulating factor-1 for 1 wk (9). More than $95 \%$ of these cells stained positive for ED-1 and were thus judged to be of a monocyte/macrophage lineage. Culture medium was replaced with medium not containing macrophage colony stimulating factor-1 24 $\mathrm{h}$ before stimulation. Rat peritoneal neutrophils were obtained by casein elicitation according to a protocol originally described for Guinea pigs, except that $9 \%$ casein was used (16). Aortic smooth muscle cells derived from Sprague-Dawley rats were the generous gift of Masao Yoshizumi (Harvard School of Public Health, Boston, MA). Murine and human cell lines were obtained from the American Type Culture Collection (Rockville, MD).

Cloning. A 3 ' partial AIF-1 cDNA fragment (382 bp) was identified previously by differential mRNA display on the basis of increased expression in allogeneic hearts compared with syngeneic hearts (12). This partial $3^{\prime}$ cDNA fragment was then used to screen $\sim 200,000$ plaques from a bacteriophage lambda, Uni-ZAP cDNA library prepared from 14-d cardiac allograft poly $(A)^{+}$RNA (Stratagene Inc., San Diego, CA). Positive clones were isolated and rescued as plasmids, and their identity was verified by demonstration of allograft-specific hybridization on Northern blot analysis. DNA sequencing of both sense and antisense strands was performed with the Sequenase 2.0 kit (United States Biochemical, Cleveland, $\mathrm{OH}$ ) on double-stranded plasmid DNA clones and subclones. Nucleotide and predicted amino acid sequence searches of the GenBank (release 84.0) and EMBL (release 36.0) databases were performed with the GCG software package, and motif searches were completed with the Prosite (release 92) data base. A hydrophilicity profile was generated by using the MacVector software and the KyteDoolittle algorithm.

Northern and genomic Southern analysis. Total RNA was isolated with RNAzol B (Tel-Test, Friendswood, TX). Northern blots were prepared with $20 \mu \mathrm{g}$ of total RNA as described (12). Genomic DNA was isolated and digested with the indicated restriction endonucleases, electrophoresed through $0.8 \%$ agarose gels, and transferred to nitrocellulose (Micron Separations, Westboro, MA) by capillary transfer as described (17). Hybridization with cDNA probes labeled with ${ }^{32} \mathrm{P}$ dCTP by random priming (Boehringer-Mannheim Corp., Indianapolis, IN) and high-stringency washes $\left(0.2 \times \mathrm{SSC}, 0.1 \% \mathrm{SDS}\right.$ at $\left.60^{\circ} \mathrm{C}\right)$ were performed as described (12).

Semiquantitative reverse-transcriptase PCR assay. To conserve RNA, we used a modified reverse-transcriptase PCR technique to study AIF-1 transcript levels instead of Northern blot analysis $(9,18)$. These reverse-transcriptase PCR assays are sensitive, require only $2.5 \mu \mathrm{g}$ total RNA, and permit replicates to be performed from a single cDNA preparation (18). cDNA synthesis was completed with random primers (2.5 $\mu \mathrm{g}$ of total RNA per reaction). Oligonucleotides were synthesized by Genosys Biotechnologies, Inc. (The Woodlands, TX). To amplify rat cDNAs we used the $5^{\prime}$ primer GTCAATTCGCTATGAGCCAGAGCAAG and the ${ }^{\prime}$ ' primer GAAGAAGCAGTTGTGAGCGTCGACCAA, a combination that resulted in a 543-bp product. For murine and human cDNAs we used an internal set of primers, 5' ATCCCAAGTACAGCAGTGATGAGG and $3^{\prime}$ GTCCCCCAGCCAAGAAAGCTATTT, which generated a 329-bp PCR product. Reaction conditions were as described (9). The thermal cycling parameters were denaturation at $94^{\circ} \mathrm{C}$ for $15 \mathrm{~s}$, annealing at $56^{\circ} \mathrm{C}$ for $20 \mathrm{~s}$, and extension for $60 \mathrm{~s}$ (with a final extension of $7 \mathrm{~min}$ at the end of all cycles). For quantitative PCR analyses, $150,000 \mathrm{cpm}$ of ${ }^{32} \mathrm{P}$-dCTP were included in the PCR reaction. The products were separated on $1 \%$ agarose gels, which were dried and exposed to PhosphorImaging screens for $24 \mathrm{~h}$, and the amount of ${ }^{32} \mathrm{P}$ incorporated in the amplified product bands was measured by volume integration (Imagequant software; Molecular Dynamics, Sunnyvale, CA).
To identify the optimal PCR conditions for accurate measurement of gene transcript levels, we established the linear assay ranges with respect to cycle number and starting template concentration for various dilutions of cDNA. Measurement of AIF-1 transcript levels was then completed within these ranges ( 23 cycles and cDNA dilutions of 1.25 $\mu l$ for both primer combinations). PCR analyses were completed on each set of cDNA at least three times. PCR amplification with G3PDH, a ubiquitously expressed gene, was used as a control to assess variations in total RNA or cDNA loading between samples. Corrected AIF-1 values were derived by dividing the measured amplified product value by the mean of the G3PDH value obtained for that cDNA from at least three analyses. Results were subjected to multivariate analysis of variance without replication (10). If a difference was significant, individual comparisons were corrected by the Bonferroni method. Although there were variations in absolute values derived from different experiments, relative differences between cDNA sets analyzed at the same time were preserved.

In situ hybridization. In situ hybridization was completed as described (19), using 5- $\mu \mathrm{m}$ frozen sections obtained from $28-\mathrm{d}$ cardiac allografts and paired host hearts and spleens. To generate radiolabeled antisense and sense transcripts, we linearized the full-length AIF-1 cDNA and transcribed it with $\mathrm{T} 7$ or $\mathrm{T} 3$ polymerase in the presence of ${ }^{35}$ S-UTP. The specificity of the riboprobes was confirmed by Northern analysis. The antisense probe hybridized to $0.7-\mathrm{kb}$ transcripts in lanes with cardiac allograft total RNA but not in lanes with day- 0 heart total RNA. The sense probe did not hybridize.

Protein expression studies. Recombinant AIF-1 was generated as a fusion protein using the pMAL-c2 (New England Biolabs Inc., Beverly, MA) or the pFLAG-1 (International Biotechnologies, Inc., New Haven, CT) expression vector according to the manufacturers' instructions. The purified protein was used as an immunogen to generate a polyclonal rabbit antiserum (East-Acres Biologicals, Southbridge, MA). Tissue or cellular extracts were prepared by homogenization in $50 \mathrm{mM}$ Tris ( $\mathrm{pH}$ 8.0), $500 \mathrm{mM} \mathrm{NaCl}, 20 \%$ glycerol, $1 \mathrm{mM}$ DTT, and $0.5 \mathrm{mM}$ PMSF. To determine whether AIF-1 was present in conditioned supernatant, we concentrated the proteins in the supernatant by ultrafiltration (Microcon 10 ultraconcentrator; Amicon, Beverly, MA). Protein extracts (25 $\mu \mathrm{g})$ were separated on $15 \%$ SDS-polyacrylamide gels and transferred to polyvinylidene difluoride membranes (Millipore Corp., Bedford, MA). Immunostaining was performed with an enhanced chemoluminescence detection kit (ECL; Amersham Corp., Cleveland, $\mathrm{OH}$ ) according to the manufacturer's directions, at primary antiserum dilutions of 1:3,000. Negative controls included omission of the primary antibody or use of preimmune serum. Immunohistochemical staining of frozen sections from 28-d cardiac allografts, host hearts, and spleens was completed at an antiserum dilution of 1:500 $\left(1 \mathrm{~h}, 25^{\circ} \mathrm{C}\right)$ as described (9). Negative controls included omission of the primary antiserum, use of preimmune serum, and use of an irrelevant primary antiserum.

In vitro and in vivo regulation of AIF-1 transcript levels. In vitro stimulation studies of various macrophage cells or cell lines were completed using rat IFN- $\gamma(100 \mathrm{U} / \mathrm{ml})$ (GIBCO BRL, Gaithersburg, MD), phorbol 12-myristate-13-acetate $(50 \mathrm{ng} / \mathrm{ml})$ (Sigma), or LPS (100 ng/ ml) (Escherichia coli serotype 0111:B4; Sigma). To study whether strategies that impair T cell activation in vivo would alter AIF-1 transcript levels, we evaluated cDNA samples from two series of transplants performed previously. In the first series the cDNAs were prepared from transplanted and host hearts harvested at day 7 and 28 from recipients fed an anti-inflammatory, essential fatty acid-deficient (EFAD) diet or a control diet (13). In the second transplant series the recombinant fusion protein CTLA-4 Ig (Bristol-Myers Squibb, Seattle, WA), which blocks the CD28/B7 costimulatory pathway, was used to inhibit $\mathrm{T}$ cell activation in chronic cardiac rejection (14). These cDNA samples were prepared from 75-d cardiac allografts treated with $(a)$ a single dose of CTLA-4 Ig, which reduces mononuclear cell infiltration $(0.5 \mathrm{mg}$ intraperitoneally, $n=3)$, (b) a single dose of CTLA-4 Ig (0.5 mg intraperitoneally) combined with an intravenous injection of donor splenocyte cells, which almost completely eliminates mononuclear cell infiltration $(n=3)$, or $(c)$ a single dose of cyclosporine $A$, which is associated with significant mononuclear cell infiltration $(5 \mathrm{mg} / \mathrm{kg}$ 
A

GAGGAGCCAGCCAACACACTGCAGCCTCATCGTCATCTCCCCACCTAAGGCCACCAGCGT CTGAGGAGCTATGAGCCAGAGCAAGGATTTGCAGGGAGGAAAAGCTTTTGGACTGCTGAA 120

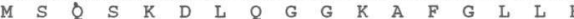

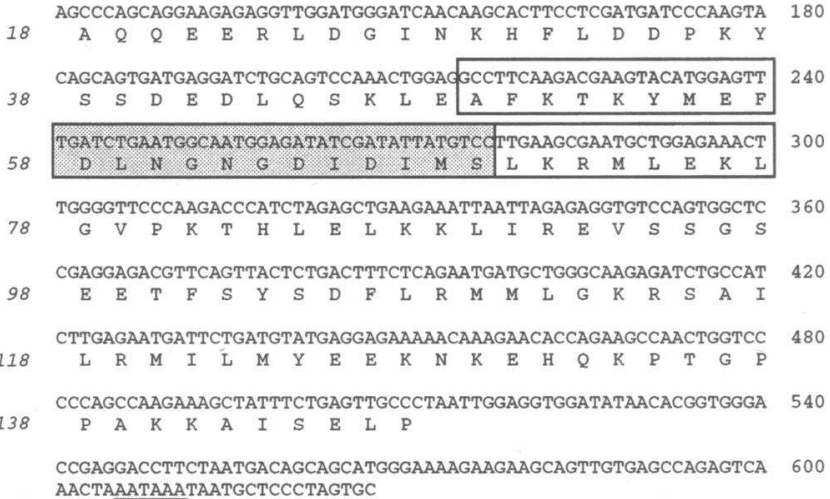

$\mathbf{B}$

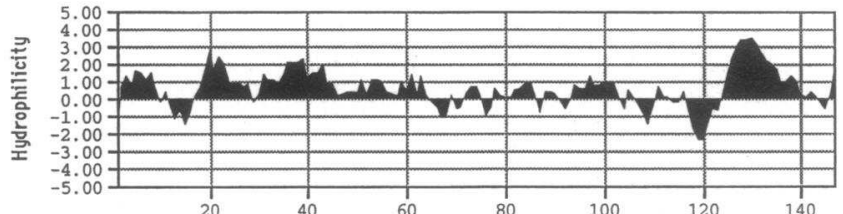

Figure 1. Nucleotide sequence of AIF-1 cDNA. (A) Nucleotide sequence and deduced amino acid sequence of the AIF-1 cDNA. Nucleotide numbering is indicated on the right and amino acid numbering is indicated on the left. The boxed area indicates the region of the EFhand-like motif (with the conserved loop segment shaded). A putative polyadenylation site is underlined. $(B)$ The AIF-1 hydrophilicity plot was generated with the Kyte-Doolittle algorithm. Regions of hydrophilicity are above the center line. These sequence data are available from EMBL/Genbank/DDBJ under accession number U17919.

intramuscularly; Sandoz, Basel, $n=3$ ). These samples were compared with samples from day-75 syngrafts $(n=3)$ and an untreated (reference) day-75 cardiac allograft $(n=1)$.

\section{Results}

Isolation and characterization of full-length AIF-1 cDNA clones. We used a previously identified 3' AIF-1 cDNA fragment (382 bp) to screen a rat cardiac allograft cDNA library and isolated eight identical clones. The full-length cDNA (Fig. $1 \mathrm{~A})$ was $627 \mathrm{bp}$ long and contained a 70-bp 5' untranslated region, a continuous open reading frame of $441 \mathrm{bp}$, and a 116bp 3' untranslated region that included one potential polyadenylation sequence. Translation of the open-reading frame predicted a polypeptide of 147 amino acids, with a calculated molecular mass of $16.8 \mathrm{kD}$. Charged amino acids comprised $35 \%$ of the predicted polypeptide. The profile generated by the KyteDoolittle algorithm (Fig. $1 B$ ) predicted a hydrophilic polypeptide without significant hydrophobic stretches, which are usually associated with membrane-spanning proteins.

Database searches revealed homology with genomic sequences obtained from a $90-\mathrm{kb}$ segment of the human HLA class III region (which had been systematically sequenced in a mapping effort) (20). Homology ranged from 68-93\% over the entire AIF-1 cDNA sequence. Alignment was identified in a segment $7 \mathrm{~kb}$ upstream of the BAT- 2 gene. To date no coding sequences have been submitted to the GenBank and EMBL data

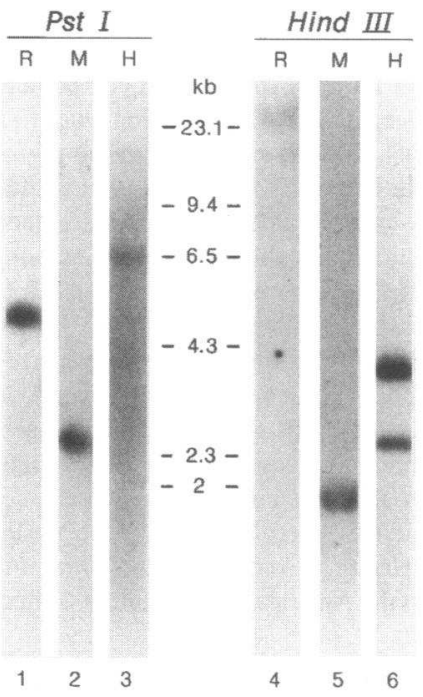

Figure 2. Genomic Southern analysis. Representative autoradiogram from a blot hybridized with the ${ }^{32} \mathrm{P}$-labeled rat AIF-1 cDNA. Genomic DNA extracted from rat $(R)$, mouse $(M)$, and human $(H)$ tissue samples was digested with the restriction enzymes indicated above the blots. bases in this region; thus, AIF-1 appears to represent a novel cDNA. Motif analysis of the predicted amino acid sequence identified a potential EF-hand domain that is characteristic of an evolutionary family of calcium-binding proteins (21). Typically, the conserved amino acids within the loop are involved in binding of the calcium ion. AIF-1 matches the consensus sequence of the EF-hand loop supplied through the Prosite data base (release 9.2), except at position 12. This conserved position-12 residue (usually glutamic acid or aspartic acid) is replaced by a serine residue in the AIF-1 sequence. In addition to the conserved loop sequence, AIF-1 has some alignment within the entire EF-hand region to other members of the family, such as mouse troponin C (51\% similar or identical amino acids) (22) and human calmodulin (48\% similar or identical amino acids) (23).

The AIF-1 cDNA hybridized to genomic DNA from rats, mice, and humans (Fig. 2), demonstrating evolutionary conservation across these species. The presence of only one or two bands under high-stringency conditions suggests that AIF-1 is encoded by a single-copy gene.

Northern blot analysis of rat organs. Northern blot analysis (Fig. 3) showed strong hybridization of the AIF-1 cDNA to 0.7-kb transcripts in total RNA from a day-28 cardiac allograft, spleen, and testis. The increase in AIF-1 transcripts in this cardiac allograft in comparison with a control heart (day 0 ), which had been harvested but not transplanted, confirms the allograftspecific pattern identified in our previous studies with partial cDNAs (12). The presence of AIF-1 transcripts in the spleen (a major source of inflammatory cells) as well as in the cardiac allograft - whose vessels are characterized at day 28 by mononuclear cell infiltration and early intimal thickening - suggests that AIF-1 represents an inflammatory cell transcript. (The expression in testis has been extensively studied but is the subject of another report.)

AIF-1 gene expression increases in cardiac allografts over time. To study AIF-1 gene expression patterns after cardiac transplantation, we measured relative transcript levels with a reverse-transcriptase PCR assay which requires 10 -fold less RNA and permits analysis of replicates. cDNAs derived from cardiac allografts were compared with those from paired host hearts harvested at various stages of chronic rejection. As seen in the representative analysis that included two transplants from 


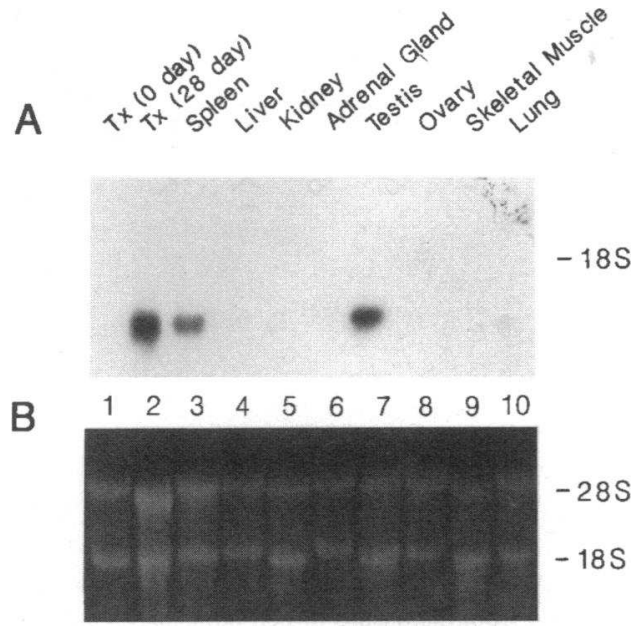

Figure 3. Northern blot analysis of rat organs. (A) Representative autoradiogram from a blot containing total RNA extracted from the indicated rat organs after hybridization with ${ }^{32} \mathrm{P}$-labeled AIF-1 cDNA. AIF-1 transcripts of $0.7 \mathrm{~kb}$ are visible in the 28-d cardiac allograft (lane 2), the spleen (lane 3), and the testis (lane 7). (B) RNA gel stained with ethidium bromide before transfer to demonstrate loading in each lane.

each time point (Fig. 4), AIF-1 transcript levels were significantly higher in cardiac allografts (days $7,28,75$ ) in comparison with paired host hearts, which had been exposed to the same circulation but had no histologic abnormalities $(P<0.003)$. Transcript levels were also significantly higher in day-75 cardiac allografts $(n=2)$ compared with day-75 cardiac syngrafts $(n=2)(P<0.0001)$. AIF-1 transcript expression peaked at day 28 , with cardiac allograft levels significantly higher in comparison with those at both days 7 and $75(P<0.001)$ in two separate experimental sets. These findings confirm the allograft-specific and time-dependent expression of AIF-1 transcripts originally observed in Northern blot studies in which the AIF-1 3' cDNA fragment had been used as probe (12).

In situ localization of AIF-1 mRNA to inflammatory infil-

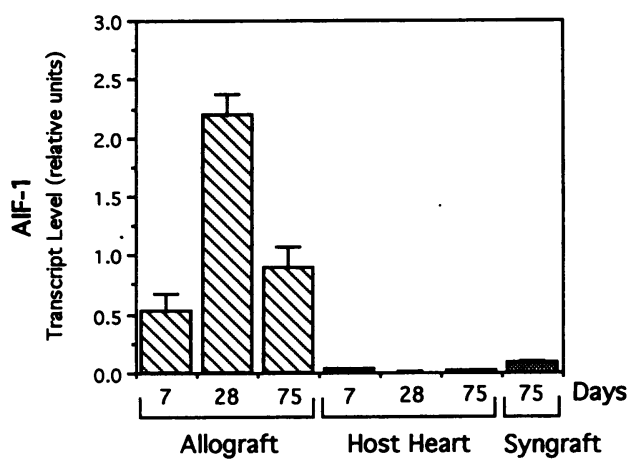

Figure 4. Time course of AIF-1 gene expression after allogeneic cardiac transplantation. Corrected levels were derived by normalizing AIF-1 ${ }^{32} \mathrm{P}$-reverse-transcriptase PCR values against those for the control gene, G3PDH, and are shown in relative units. Levels increased significantly in cardiac allograft cDNA at 7, 28, and $75 \mathrm{~d}$ after transplantation (hatched bars) compared with cDNA from paired host hearts (black bars $)$ and day-75 syngrafts (stippled bar $)(P<0.003)$. Values are shown for a representative experiment on two transplants per group. Data are plotted as means \pm SEM and represent three separate PCR analyses.

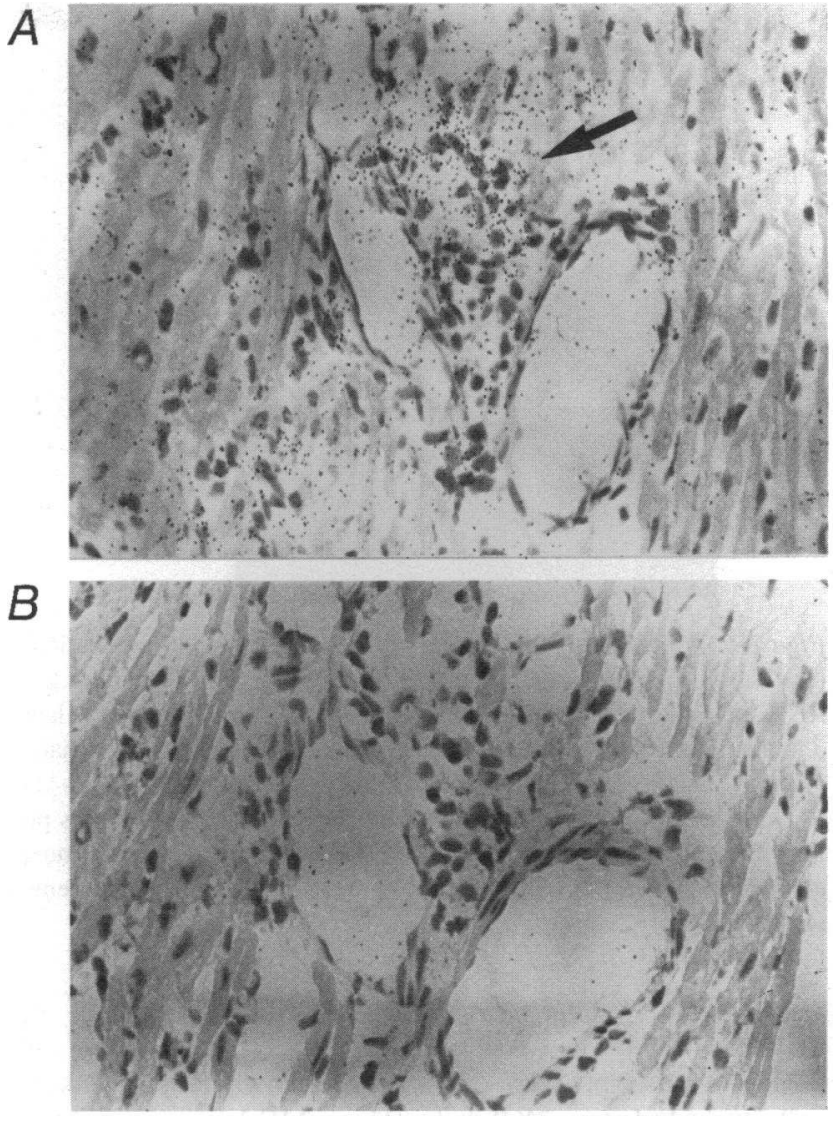

Figure 5. In situ localization of AIF-1 transcripts in rat cardiac allografts. Frozen sections were hybridized with ${ }^{35} \mathrm{~S}$-UTP-labeled antisense ( $A$ $\times 300)$ and sense $(B \times 300)$ riboprobes. Silver grains indicating hybridization of AIF-1 mRNA are clustered over mononuclear inflammatory cells in the perivascular (arrow) and interstitial spaces.

trates within cardiac allografts. In Fig. 5 A silver grains indicating hybridization of the AIF-1 antisense riboprobe are seen to cluster over regions of inflammatory cell infiltrates in cardiac allograft sections, and they do not cluster over myocytes. Positive hybridization was predominately in the interstitium and perivascular spaces. In splenic tissue, silver grains associated with AIF-1 mRNA were identified primarily in regions within the red pulp (not shown). There was no significant hybridization to host hearts (not shown) or when the sense probe was used (Fig. $5 \mathrm{~B}$ ). In sections from rat and mouse testis, silver grains associated with AIF-1 mRNA were concentrated over differentiated germ cells in the seminiferous tubules (not shown).

AIF-1 transcript levels in inflammatory cell populations. To further characterize the cell type(s) that express AIF-1 transcripts, we analyzed a series of isolated rat cell populations by Northern blotting (Fig. 6, $A$ and $B$ ). AIF-1 transcripts were expressed by bone marrow-derived macrophages, bacille $\mathrm{Cal}$ mette Guérin-elicited peritoneal macrophages, and casein-elicited peritoneal neutrophils but not significantly by nylon woolpurified lymphocytes, aortic smooth muscle cells, or bone marrow stem cells. In other studies (not shown), AIF-1 transcripts were detected in thioglycolate-elicited peritoneal macrophages and adherent splenocytes. By the reverse-transcriptase PCR assay, AIF-1 transcripts were also present at low levels in a variety of unstimulated murine and human myeloid cell lines 

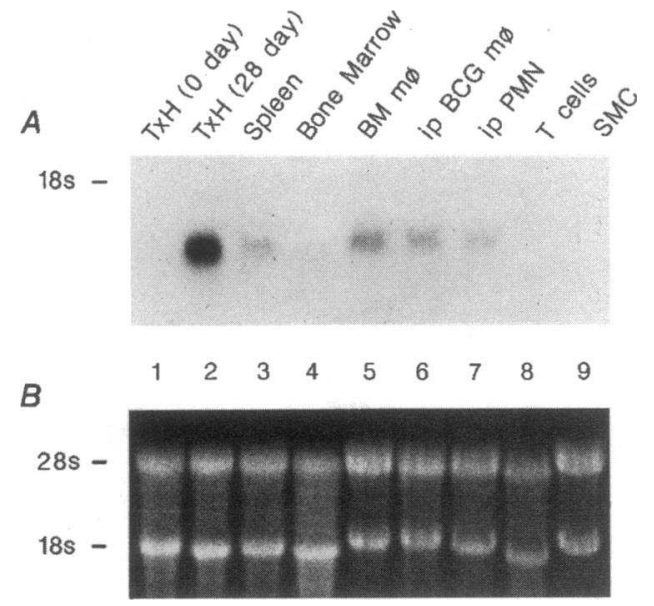

Figure 6. AIF-1 transcript expression in isolated rat cell populations. (A) Representative autoradiogram of a Northern blot containing total RNA extracted from cardiac allografts (lanes 1 and 2), spleen (lane 3 ), bone marrow cells (lane 4), macrophages derived from bone marrow (lane 5), bacille Calmette Guérin-elicited peritoneal macrophages (lane 6), casein-elicited peritoneal neutrophils (lane 7), T lymphocytes purified from a splenocyte suspension (lane 8 ), and aortic smooth muscle cells (lane 9). (B) RNA gel stained with ethidium bromide to demonstrate loading.

including J774A.1, RAW264.7, P388D.1, HL60, U937, and THP-1. Transcripts were not detected in lymphocytic leukemia cell lines (Jurkat or MOLT-4). Thus, AIF-1 appears to be expressed predominately by cells of the myeloid lineage (monocytes/macrophages and neutrophils).

Immunoblotting with anti-AIF-1 serum. A single band of $\sim 17 \mathrm{kD}$ was detected with polyclonal rabbit anti-AIF-1 serum in extracts from cardiac allografts, splenic tissue, and bone marrow-derived macrophages propagated in vitro (Fig. 7). However, AIF-1 was not detected in extracts from the conditioned supernatant of resting or IFN- $\gamma$-stimulated bone marrow-derived macrophages or J774A.1 cells (not shown), demonstrating that it is not released into the media. The presence of AIF1 protein in cardiac allografts and its absence in host hearts, lymphocytes, and bone marrow cells (by immunoblotting) reproduced the patterns shown for gene transcripts.

Immunohistochemical localization of AIF-1 antigen in cardiac allografts. Immunostaining identified a subset of positively labeled mononuclear cells within cardiac allografts (Fig. $8 \mathrm{~A}$ ). The positive cells showed a cytoplasmic staining pattern and were found in regions of perivascular and interstitial infiltration. Besides these inflammatory mononuclear cells, none of the parenchymal cell types within the cardiac allograft stained positive. In splenic tissue scattered positive cells were identified predominately in the red pulp (Fig. $8 B$ ). There was also a rim of positive cells just adjacent to the white pulp. In testis the AIF-1 antigen localized predominately to germ cells and occasionally to monocyte/macrophages (not shown), suggesting that it may play a distinct role in this tissue-perhaps in germ cell differentiation. In all cases, no significant staining was seen when the primary antibody was omitted or when preimmune serum was used.

In vitro upregulation of AIF-1 transcripts in IFN- $\gamma$-treated macrophages. We have recently shown that the expression of the T cell-derived cytokine IFN- $\gamma$ is persistently upregulated in chronically rejecting cardiac allografts (14). To examine
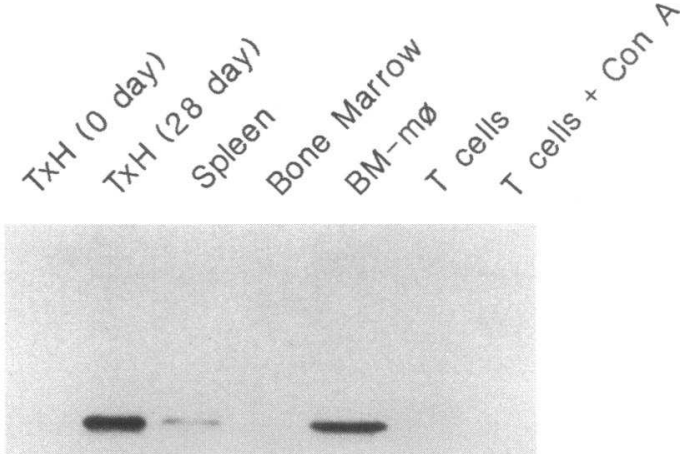

Figure 7. Detection of AIF-1 antigen on immunoblots. Western blots containing protein extract from the tissues or cell populations indicated were immunostained with rabbit anti-AIF-1 serum. Chemoluminescent detection shows a band of $17 \mathrm{kD}$ in extracts from a day-28 cardiac allograft (lane 2), spleen (lane 3 ), and bone marrow-derived macrophages (lane 5) but not in extracts from a host heart (lane 1 ), femoral bone marrow cells (lane 4 ) or unstimulated (lane 6 ) or Concanavalin A-stimulated T lymphocytes (lane 7).

whether IFN- $\gamma$ is involved in the regulation of AIF-1 expression, we performed in vitro stimulation studies with various rodent macrophage-like cell lines or isolated macrophage populations. In these studies IFN- $\gamma$ stimulation increased AIF-1 mRNA expression in mouse J774A.1, RAW264.7, and P388D.1 cell lines, as well as in rat bone marrow-derived macrophages. The basal level of AIF-1 expression varied among the cell populations, as did the peak expression level in response to IFN- $\gamma$ stimulation (three- to sixfold). In a representative study of J774A.1 cells (Fig. 9 A), AIF-1 transcript levels increased significantly (fivefold) $24 \mathrm{~h}$ after stimulation with IFN- $\gamma$ in comparison with cells stimulated with phorbol 12-myristate-13-acetate or LPS $(P<0.0001)$. In contrast, the addition of LPS antagonized IFN- $\gamma$-induced expression of AIF-1, as has been reported for other macrophage factors $(24,25)$. AIF-1 transcript levels did not change significantly at 4 and $10 \mathrm{~h}$ with any of the stimulants studied. A more detailed time-course study of the IFN- $\gamma$ response showed that the increase in AIF-1 transcripts was delayed, peaking in J774A.1 cells at $36 \mathrm{~h}$ after stimulation (Fig. $9 B$ ). The most effective dose of IFN- $\gamma$ was $100 \mathrm{U} / \mathrm{ml}$ of medium (Fig. $9 \mathrm{C}$ ). Immunoblotting confirmed these time- and dose-dependent increases in AIF-1 expression (data not shown). This late increase in AIF-1 expression suggests that its up-regulation by IFN- $\gamma$ may be an indirect effect.

In vivo modulation of $T$ cell activation reduces AIF-1 transcripts in cardiac allografts. To study the regulation of AIF-1 expression in vivo we applied strategies that attenuate $T$ cell activation and reduce IFN- $\gamma$ expression. Modulation of the inflammatory response with an EFAD diet decreases expression of IFN- $\gamma$ at day 28 , reduces mononuclear cell infiltration, and attenuates arteriosclerotic development in Lewis to F344 cardiac allografts (13). Here we show that AIF-1 transcript levels in 28-d cardiac allografts on the EFAD diet were reduced significantly in comparison with those in allografts from recipients fed a control diet $(P<0.0001)$ (Fig. $10 A)$. In contrast, at day 7 (before the EFAD diet has its effect), there was no significant difference in AIF-1 transcript levels in allografts on the EFAD diet versus the control diet. AIF-1 transcript levels in host hearts were uniformly low. 

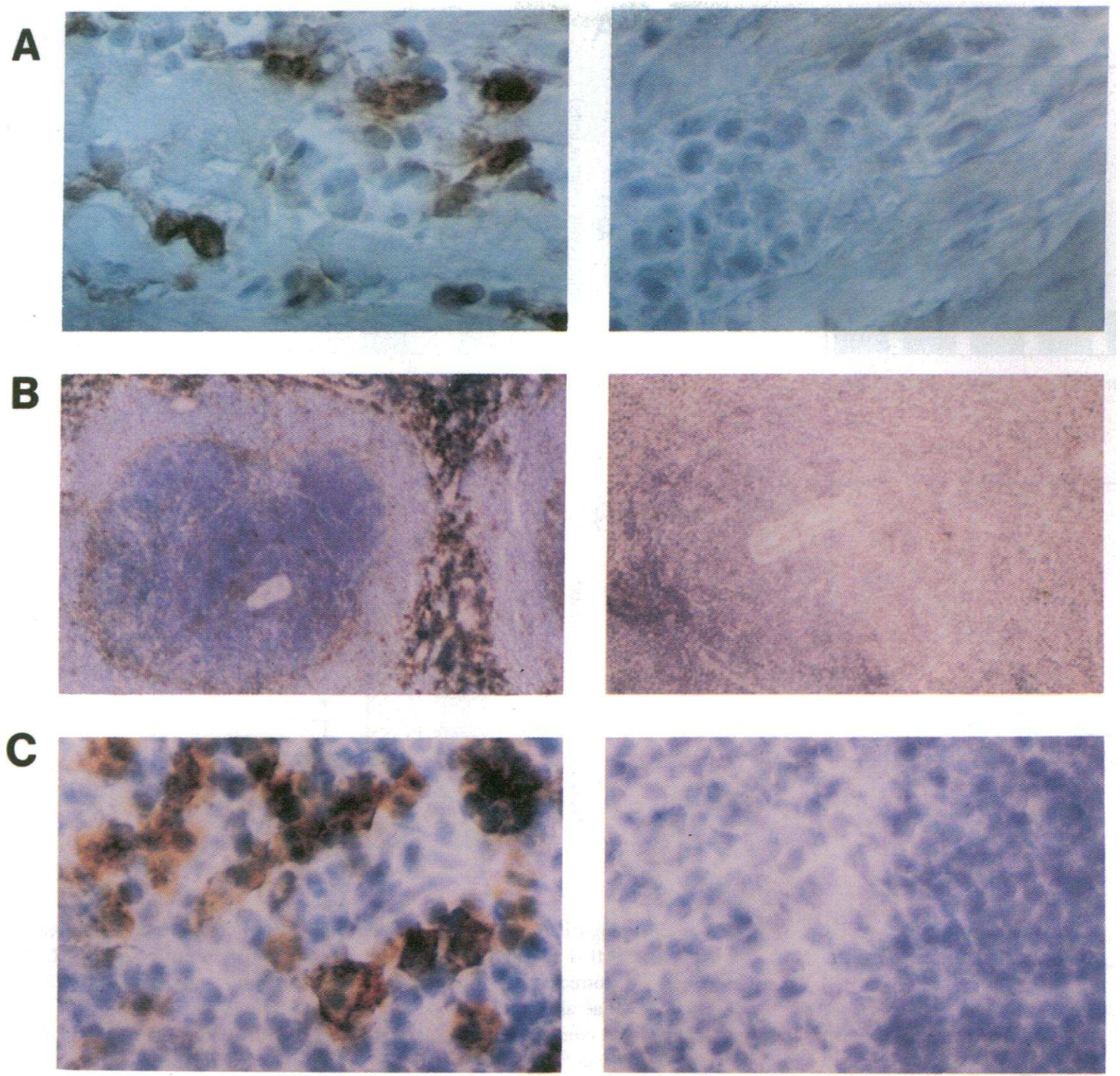

Figure 8. Immunostaining of the AIF-1 antigen in tissue sections. Cells showing AIF-1-positive staining in the cytoplasm of a subset of mononuclear cells (left panels) are visible in the interstitium of a 14-d cardiac allograft $(A \times 940)$ and in the splenic red pulp $(B \times 80$ and $C \times 940)$. No staining is visible if the primary antibody is omitted (right panels).

Preliminary studies from our laboratory indicate that blocking the CD28/B7 costimulatory pathway of $\mathrm{T}$ cell activation with CTLA-4 Ig prolongs allograft survival, reduces mononuclear cell infiltration, and prevents $\mathrm{T}$ cell and macrophage activation in the Lewis to F344 rat model (14). AIF-1 transcript levels were reduced significantly in cardiac allografts from recipients treated with CTLA-4 Ig in comparison with untreated allografts or cyclosporine A-treated (single dose on day 2) allografts $(P<0.0001)$ (Fig. $10 B)$. The most dramatic decrease was seen in the group that received CTLA-4 Ig in addition to donor cells on the day of engraftment. AIF-1 transcript levels in this group were significantly lower than those in cardiac allografts that received CTLA-4 Ig alone $(P<0.0001)$ and similar to those seen in control syngrafts. The decrease in AIF1 expression in cardiac allografts treated with the EFAD diet and with CTLA-4 Ig corresponded to a reduction in the number of ED-1-positive macrophages. Thus, in cardiac allografts treated with CTLA-4 Ig there was a decrease in AIF-1 transcripts that reflected the decrease in macrophage density. How- ever, a direct or indirect effect of CTLA-4 Ig on AIF-1 expression may also contribute.

\section{Discussion}

Here we report the cloning of AIF-1 cDNA and the characterization of its expression in vivo and in vitro. The $3^{\prime}$ portion of the AIF-1 cDNA was originally identified in a screen for differentially expressed genes present in cardiac allografts compared with reference syngrafts (12). The strategy was to analyze cDNAs from the cardiac allografts directly, as a way of preserving the infiltrating macrophages and the allogeneic microenvironment that regulated their functional state. Our further characterization of AIF-1 has demonstrated that it is a novel factor selectively expressed by inflammatory cells of the monocyte/ neutrophil lineage and up-regulated by IFN- $\gamma$, although not necessarily specific for allogeneic inflammation. The high levels of AIF-1 in allografts reflected in part the high density of infil- 
A

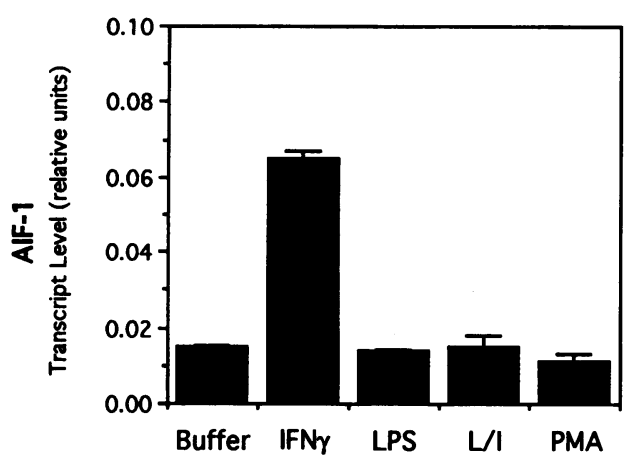

B

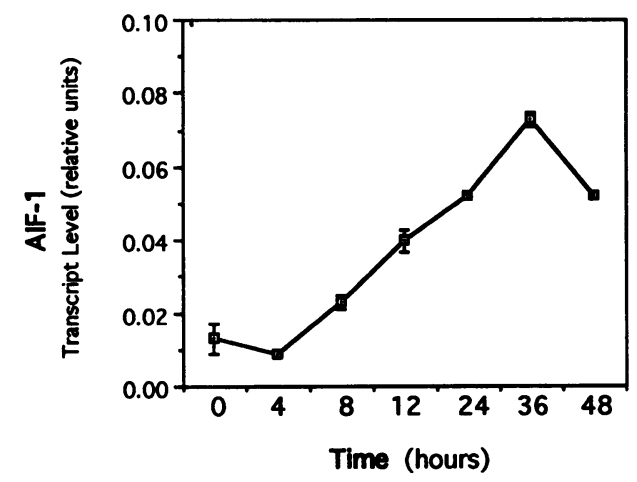

C

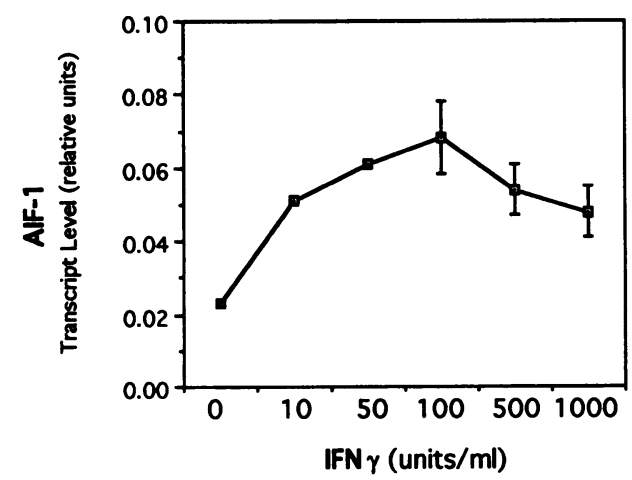

Figure 9. Upregulation of AIF-1 transcript levels by IFN- $\gamma$ in vitro. $(A)$ Corrected AIF-1 transcript levels were significantly higher in IFN$\gamma$-treated J774A.1 cells $(P<0.0001)$ compared with cells treated with buffer, LPS, combined LPS and IFN- $\gamma(L / I)$, or phorbol 12-myristate13-acetate $(24 \mathrm{~h})$. (B) Time-dependent increase in AIF-1 transcript levels in J774A.1 cells cultured in the presence of $100 \mathrm{U} / \mathrm{ml} \mathrm{IFN-} \gamma$ for the indicated periods. $(C$ ) Dose-response in J774A.1 cells of IFN- $\gamma$ induced increases in AIF-1 transcript levels after $24 \mathrm{~h}$ of stimulation. Data are plotted as means \pm SEM and represent three separate PCR analyses. The data are representative of three independent experiments.

trating macrophages and the cytokine-rich environment which may up-regulate AIF-1 expression.

The full-length AIF-1 cDNA represents a novel coding sequence that is conserved among the mouse, rat, and human species. The AIF-1 gene appears to be linked to the human
A

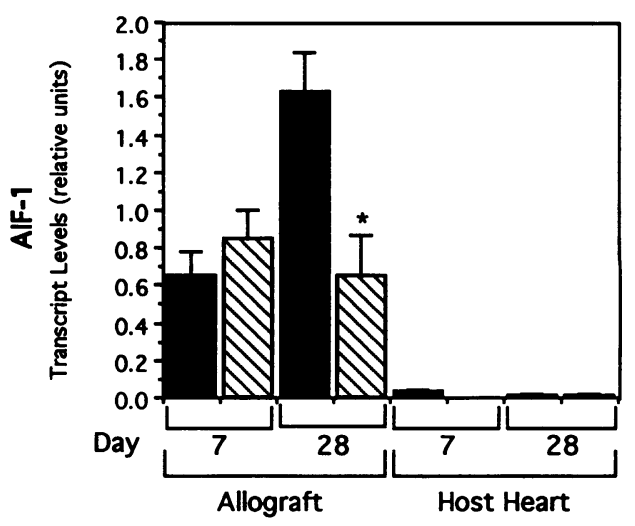

B

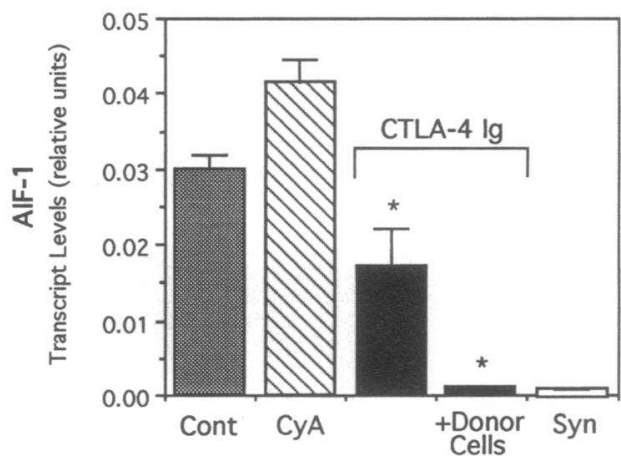

Figure 10. Modulation of the allogeneic immune response decreases AIF-1 transcript levels in cardiac allografts. $(A)$ Effect of EFAD diet. Corrected AIF-1 transcript levels decreased significantly in day-28 cardiac allografts harvested from rats fed the EFAD diet (hatched bars) in comparison with allografts harvested from rats fed a control diet (black bars) $(P<0.0001)$. (B) Effect of CTLA-4 Ig. Corrected AIF1 transcript levels were significantly lower in cardiac allografts harvested at day 75 from rats treated with CTLA-4 Ig (black bar) $(P<0.0001)$ in comparison with allografts from control rats (stippled bar) or allografts from cyclosporine A-treated animals (hatched bar). Administration of CTLA-4 Ig in combination with donor cells further decreased AIF-1 transcripts to a level similar to that observed in syngrafts (white bar $)(P<0.0001)$. Data are plotted as means \pm SEM from two or three transplants per group and represent three separate PCR analyses.

HLA class III region because it aligns with genomic sequences in a $90-\mathrm{kb}$ segment that had been sequenced in a systematic mapping effort (20). However, no coding sequences have been reported to date in the homologous region. The presence of an EF hand-like motif within the AIF-1 amino acid sequence is intriguing. Two or more EF-hand domains are typical of the family of intracellular calcium-binding proteins that includes calmodulin and troponin C (26). However, AIF-1 deviates from this family because it contains only a single EF-hand and, in spite of the overall conservation in the EF-hand domain, it lacks a conserved amino acid residue required for $\mathrm{Ca}$ binding. Thus, the relation of AIF-1 to the EF-hand family remains to be established.

Analysis of AIF-1 gene transcript and product expression confirmed and extended our original finding that it is present in rat cardiac allografts undergoing chronic rejection but absent 
in syngrafts and paired host hearts (exposed to the same circulation but with normal vessels) (12). More extensive studies completed with a series of cardiac transplants harvested at various time points showed that AIF-1 transcripts increased significantly in allografts by day 7 and remained elevated through days 28 and 75. In contrast, AIF-1 levels were low in paired host hearts. This early and sustained expression of AIF-1 is consistent with an ongoing allogeneic inflammatory stimulation, as might be expected in chronic rejection.

In cellular localization studies performed with cardiac allograft and splenic tissue sections, AIF-1 localized to mononuclear cells and was found predominantly in regions occupied by macrophages. In cardiac allografts, ED-1-positive macrophages have been shown to be the predominant infiltrating cell type in the interstitium and perivascular space (7). Hence, the AIF-1-positive cells described here are most likely macrophages. In the spleen AIF-1 staining was found in the red pulp within a subset of cells with macrophage features, and in cells forming a rim in the white pulp typical of marginal macrophages (27). In contrast, in the testis there was a very high level of expression of AIF-1 mRNA and antigen by differentiating germ cells (data not shown), suggesting that AIF-1 has a distinct role in spermatogenesis as opposed to inflammation. In vitro studies of isolated cells and cell lines from rats, mice, and humans also confirmed the selective expression of AIF-1 by cells of the monocyte/macrophage lineage, in comparison with cells of the lymphoid lineage. AIF-1 was also expressed by elicited neutrophils, a cell type seen infrequently in cardiac allografts and spleen.

Myeloid specificity is a feature shared by other members of the EF-hand family. The best studied examples are MRP8/ MRP14, which have been associated with a number of acute and chronic inflammatory diseases (28), and murine CP-10, a chemotactic protein for neutrophils and monocytes (29). Like AIF-1, these proteins lack classic signal sequences. This lack raises questions about whether they attain extracellular bioactivity at cell death or through alternative transport mechanisms. Studies in vitro and in vivo have shown that the hinge region between the two EF-hands of CP-10 contributes significantly to its chemotactic activity (30). Further studies will be needed to determine whether AIF-1 functions in the recruitment of cells to sites of injury.

We also studied the regulation of AIF-1 expression in vitro and in vivo. We have shown that a number of inflammatory mediators associated with $\mathrm{T}$ cell activation (IFN- $\gamma$ ) and macrophage activation (monocyte chemoattractant protein-1 and inducible nitric oxide synthase) are up-regulated in Lewis to F344 rat cardiac allografts undergoing chronic rejection $(8,10,18)$. IFN- $\gamma$, a cytokine secreted by activated $\mathrm{T}$ lymphocytes and natural killer cells, is a potent activator of macrophages and affects a variety of macrophage properties and functions (31). In this report we demonstrate in vitro that IFN- $\gamma$ up-regulates expression of AIF-1 in various macrophage populations (up to sixfold). The delayed increase in AIF-1 expression-it occurs 24 to $36 \mathrm{~h}$ after stimulation-suggests that the up-regulation is an indirect effect that could involve intermediate steps. Interestingly, we found that LPS antagonized the induction of AIF-1 in macrophages by IFN- $\gamma$, as has been reported for MHC class II and iNOS expression in macrophages $(24,25)$. We did observe that these same cells increased iNOS expression and nitrate production synergistically in response to the combination of LPS and IFN- $\gamma$ (data not shown), indicating that the cells can be responsive to LPS. This suggests that AIF-1 transcription might be suppressed (directly or indirectly) by LPS through another pathway that may involve a different route of signal transduction. Nonetheless, given the high expression of IFN- $\gamma$ in the interstitium and vessels of Lewis to F344 rat cardiac allografts undergoing chronic rejection (18), one can speculate that the increase in AIF-1 expression may stem in part from local up-regulation by IFN- $\gamma$ within the graft.

It has been shown that the expression of IFN- $\gamma$ in Lewis to F344 cardiac allografts is down-regulated by treatment with an EFAD diet or by blockade of T cell activation with CTLA-4 $\operatorname{Ig}(13,14)$. We examined whether these two different strategies would affect the levels of AIF-1 transcripts in vivo. The antiinflammatory effect of the EFAD diet results from defects in arachidonate metabolism and leukotriene production (which impair leukocyte chemotaxis and function) in rodent models of inflammation that include glomerulonephritis (32). In Lewis to F344 rat cardiac allografts the EFAD diet reduces mononuclear cell infiltration, the severity of intimal thickening, and the number of vessels with intimal thickening that develop at early (day $28)$ as well as late (day 120$)$ stages $(13,33)$. In this report we show that there was a significant reduction in the number of AIF-1 transcripts in day 28 cardiac allografts treated with the EFAD diet in comparison with day 28 allografts on a control diet. This reduction in AIF-1 transcripts could be due to attenuation of leukocyte infiltration or to leukocyte activation and production of cytokines such as IFN- $\gamma$, or both.

CTLA-4 Ig is a recombinant fusion protein that inhibits the CD28/B7 costimulatory pathway required for activation of lymphocytes (34). CTLA-4 Ig has been shown to prolong graft survival in rat and mouse acute rejection models $(35,36)$, but little is known about its effect on macrophages. We show here that cardiac allografts treated with CTLA-4 Ig had lower AIF1 transcript levels (correlating with a reduction in mononuclear cell infiltration) than transplants receiving the control treatment (a single dose of cyclosporine A) and untreated animals. The perioperative administration of donor cells in addition to CTLA4 Ig was associated with minimal mononuclear cell infiltration, and it resulted in a further decrease in AIF-1 transcripts, to levels similar to those seen in syngrafts. The effect of CTLA$4 \mathrm{Ig}$ alone may be incomplete, that is, it may only delay inhibition of $T$ cell activation and require repeated administration. However, with the increased availability of alloantigen supplied by donor cells it may be possible to produce a long-lasting tolerance due to clonal deletion of the alloantigen-specific $\mathrm{T}$ cell population. Our observation that expression of AIF-1 was attenuated by CTLA-4 Ig treatment in Lewis to F344 rat allografts suggests that $T$ cell activation regulates this macrophage component of the alloimmune response associated with chronic cardiac rejection.

The identification of the novel myeloid factor AIF-1 in the cytokine-rich milieu of cardiac allografts links it to the alloimmune response associated with chronic rejection and highlights the prominence of macrophages in this process. From a practical standpoint, measurement of AIF-1 transcript or expression levels may be useful in evaluating macrophage infiltration, and it may serve as a clinical marker of chronic cardiac rejection. Our in vitro studies provide evidence that AIF- 1 is a cytokineresponsive molecule that can be upregulated by IFN- $\gamma$, a product of activated $\mathrm{T}$ cells. The in vivo studies demonstrate that AIF-1 transcript levels decrease in accord with monocyte infiltration when IFN- $\gamma$ expression is attenuated in cardiac allografts treated with CTLA-4 Ig or the EFAD diet. This modulation of AIF-1 expression may be an indirect result of alterations in 
expression of cytokines or chemokines such as IFN- $\gamma$ within the graft. Alternatively, these treatments may have direct effects on AIF-1 transcription. The next step will be to determine whether the effect of these treatments is due to alterations in the degree of macrophage infiltration or impaired macrophage activation. Additional strategies for inhibiting various components of $T$ cell activation in vivo will be helpful in determining the specific factors that regulate AIF-1 expression and, ultimately, its functional role in macrophage biology.

\section{Acknowledgments}

We thank T. McVarish for his editorial assistance; M. Karnovsky for his support and permission to use transplant samples from previous collaborative work in these studies; M. Sayegh for his collaborative work involving CTLA-4 Ig; C. Geczy for helpful discussions; and E. Haber for his ongoing support and for providing a stimulating research environment.

This work was supported by a grant from Bristol-Myers Squibb.

\section{References}

1. Tilney, N. L., W. D. Whitley, J. R. Diamond, J. W. Kupiec-Weglinski, and D. H. Adams. 1991. Chronic rejection-an undefined conundrum. Transplantation. 52:389-398.

2. Schoen, F. J., and P. Libby. 1991. Cardiac transplant graft arteriosclerosis. Trends Cardiovasc. Med. 1:216-223.

3. Paul, L. C., and B. Fellstrom. 1992. Chronic vascular rejection of the heart and the kidney-have rational treatment options emerged? Transplantation. 53:1169-1179.

4. Cramer, D. V. 1993. Graft arteriosclerosis in heart transplantation. R. G. Landes Company, Austin, TX. 1-68.

5. Russell, P. S., C. M. Chase, H. J. Winn, and R. B. Colvin. 1994. Coronary atherosclerosis in transplanted mouse hearts. I. Time course and immunogenetic and immunopathological considerations. Am. J. Pathol. 144:260-274.

6. Cramer, D. V., G. D. Wu, F. A. Chapman, E. Cajulis, H. K. Wang, and L. Makowka. 1992. Lymphocytic subsets and histopathologic changes associated with the development of heart transplant arteriosclerosis. J. Heart Lung Transplant. 11:458-66.

7. Adams, D. H., L. R. Wyner, and M. J. Karnovsky. 1993. Experimental graft arteriosclerosis. II. Immunocytochemical analysis of lesion development. Transplantation. 56:794-799.

8. Russell, M. E., D. H. Adams, L. R. Wyner, Y. Yamashita, N. J. Halnon, and M. J. Karnovsky. 1993. Early and persistent induction of monocyte chemoattractant protein 1 in rat cardiac allografts. Proc. Natl. Acad. Sci. USA. 90:60866090.

9. Russell, M. E., U. Utans, A. F. Wallace, P. Liang, R. J. Arceci, M. J. Karnovsky, L. R. Wyner, Y. Yamashita, and C. Tarn. 1994. Identification and upregulation of galactose/ $\mathrm{N}$-acetylgalactosamine macrophage lectin in rat cardiac allografts with arteriosclerosis. J. Clin. Invest. 94:722-730.

10. Russell, M. E., A. F. Wallace, L. R. Wyner, J. B. Newell, and M. J. Karnovsky. Upregulation and modulation of inducible nitric oxide synthesis in rat cardiac allografts with chronic rejection and transplant arteriosclerosis. Circulation. In press.

11. Hamilton, T. A., Y. Ohmori, S. Narumi, and C. S. Tannenbaum. 1993. Regulation of diversity of macrophage activation. In Mononuclear Phagocytes in Cell Biology. CRC press, Boca Raton, FL. 47-70.

12. Utans, U., P. Liang, L. R. Wyner, M. J. Karnovsky, and M. E. Russell. 1994. Chronic cardiac rejection: identification of five upregulated genes in transplanted hearts by differential mRNA display. Proc. Natl. Acad. Sci. USA. 91:6463-6467.

13. Russell, M. E., W. W. Hancock, A. F. Wallace, L. R. Wyner, and M. J. Karnovsky. Modulation of inflammatory activation pathways in the Lewis to F344 rat chronic cardiac rejection model. Transplant Proc. In press.

14. Sayegh, M. H., E. Akalin, W. W. Hancock, and M. E. Russell. 1994 Blocking the CD28-B7 costimulatory $\mathrm{T}$ cell activation pathways with CTLA-4
Ig presents chronic rejection in the Lew to $\mathrm{F} 344$ vascularized rat cardiac allograft model [abstract]. J. Am. Soc. Nephrol. 5:989.

15. Hughes, D. A., and S. Gordon. 1993. Preparation of cells and tissues: serous cavity cells. In Methods of Immunological Analysis. VHS, Weinheim, Germany. 39-51.

16. DePierre, J. W., and M. L. Karnovsky. 1974. Ecto-enzymes of the guinea pig polymorphonuclear leukocyte. J. Biol. Chem. 249:7111-7120.

17. Russell, M. E., U. Seligsohn, B. S. Coller, M. H. Ginsberg, P. Skoglund, and T. Quertermous. 1988. Structural integrity of the glycoprotein IIb and IIIa genes in Glanzmann thrombasthenia patients from Israel. Blood. 72:1833-1836.

18. Russell, M. E., A. F. Wallace, W. W. Hancock, M. H. Sayegh, D. H. Adams, N. E. S. Sibinga, L. R. Wyner, and M. J. Karnovsky. 1995. Upregulation of cytokines associated with macrophage activation in the Lewis to F344 rat chronic cardiac rejection model. Transplantation. 59:572-578.

19. Arceci, R. J., F. Shanahan, E. R. Stanley, and J. W. Pollard. 1989. The expression of colony stimulating factor-1 (CSF-1) and its receptor in the female reproductive tract suggests a role for this growth factor in placental growth and differentiation. Proc. Natl. Acad. Sci. USA. 86:8818-8822.

20. Iris, F. J. M., L. Bougueleret, S. Prieur, D. Caterina, G. Primas, V. Perrot, J. Jurka, R.-T. P., J. M. Claverie, J. Dausset, and D. Cohen. 1993. Dense Alu clustering and a potential new member of the NF kappa B family within a 90 kilobase HLA class III segment. Nature Genet. 3:137-145.

21. Strynadka, N. C. J., and M. N. G. James. 1989. Crystal structures of the helix-loop-helix calcium-binding proteins. Annu. Rev. Biochem. 58:951-998.

22. Pamacek, M. S., A. R. Bengur, A. J. Vora, and J. M. Leiden. 1990. The structure and regulation of expression of the murine fast skeletal troponin c gene: identification of a developmentally regulated, muscle-specific transcriptional enhancer. J. Biol. Chem. 265:15970-15976.

23. Koller, M., and E. E. Strehler. 1993. Functional analysis of the promoters of the human CAMIII calmodulin gene and of the intronless gene coding for a calmodulin-like protein. Biochim. Biophys. Acta. 1163:1-9.

24. Figueiredo, F., T. J. Koerner, and D. O. Adams. 1989. Molecular mechanisms regulating the expression of class II histocompatibility molecules on macrophages. Effects of inductive and suppressive signals on gene transcription. $J$. Immunol. 143:3781-3786.

25. Bogdan, C., Y. Vodovotz, J. Paik, Q. Xie, and C. Nathan. 1993. Traces of bacterial lipopolysaccharide suppress IFN-gamma-induced nitric oxide synthase gene expression in primary mouse macrophages. J. Immunol. 151:301-309.

26. Heinzmann, C. W., and W. Hunziker. 1991. Intracellular calcium-binding proteins: more sites than insights. Trends Biochem. Sci. 16:98-103.

27. Dijkstra, C. D., E. A. Döpp, P. Joling, and G. Kraal. 1985. The heterogeneity of mononuclear phagocytes in lymphoid organs: distinct macrophage subpopulations in the rat recognized by monoclonal antibodies ED1, ED2 and ED3. Immunology. 54:589-599.

28. Hessian, P. A., J. Edgeworth, and N. Hogg. 1993. MRP-8 and MRP-14, two abundant $\mathrm{Ca}(2+)$-binding proteins of neutrophils and monocytes. J. Leukoc. Biol. 53:197-204.

29. Lackmann, M., P. Rajasekariah, S. E. Iismaa, G. Jones, C. J. Cornish, S. Hu, R. J. Simpson, R. L. Moritz, and C. L. Geczy. 1993. Identification of a chemotactic domain of the pro-inflammatory S100 protein CP-10. J. Immunol. 150:2981-2991.

30. Devery, J. M., N. J. C. King, and C. L. Geczy. 1994. Acute inflammatory activity of the S100 protein CP-10: Activation of neutrophils in vivo and in vitro. J. Immunol. 152:1888-1897.

31. Williams, J. G., G. J. Jurkovich, and R. V. Maier. 1993. Interferon- $\gamma$ a key immunomodulatory lymphokine. J. Surg. Res. 54:79-93.

32. Lef kowith, J. B., B. A. Jakschik, P. Stahl, and P. Needleman. 1987. Metabolic and functional alterations in macrophages induced by essential fatty acid deficiency. J. Biol. Chem. 262:6668-6675.

33. Adams, D. H., L. R. Wyner, M. J. Steinbeck, and M. J. Karnovsky. 1993. Inhibition of graft arteriosclerosis by modulation of the inflammatory response. Transplant Proc. 25:2092-2094.

34. Linsley, P. S., W. Brady, M. Umes, L. S. Grosmaire, N. K. Damle, and J. A. Ledbetter. 1991. CTLA-4 is a second receptor for the B cell activation antigen B7. J. Exp. Med. 174:561-569.

35. Lin, H., S. F. Bolling, P. S. Linsley, R. Q. Wei, D. Gordon, C. B. Thompson, and L. A. Turka. 1993. Long-term acceptance of major histocompatibility complex mismatched cardiac allografts induced by CTLA4 Ig plus donor-specific transfusion. J. Exp. Med. 178:1801-1806.

36. Pearson, T. C., D. Z. Alexander, K. J. Winn, P. S. Linsley, R. P. Lowry, and C. P. Larsen. 1994. Transplantation tolerance induced by CTLA4-Ig. Transplantation. 57:1701-1706. 